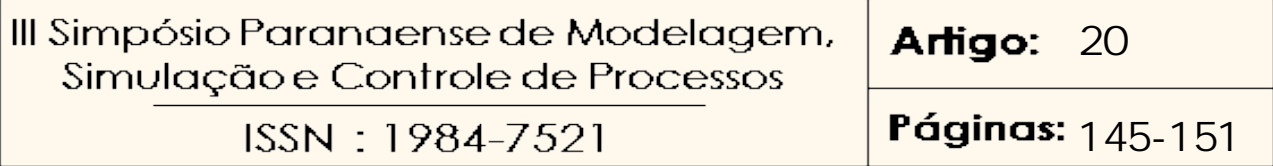

\title{
Construção e testes em um módulo experimental de um sistema de dois tanques sem interação
}

\author{
Jair G. Certório*, Cid M. G. Andrade, Camila B. Miranda \\ 1 - Universidade Estadual de Maringá -UEM - PR, jgcertorio@uol.com.br \\ 2 - Universidade Estadual de Maringá - UEM - PR \\ 3 - Universidade Estadual de Maringá - UEM - PR
}

\begin{abstract}
This paper presents the modeling and control of a low-cost experimental module for a laboratory of process control. The plant is a two tanks coupled system with the systems control being done via a DAQ. The modeling was done approximating the system as a first-order one, then, determining the black box and white box models. Also, the process was done varying parameters in the system to compare the resulting poles, which was done changing the orifice in the tank being modeled. After, PI controllers were designed to control the plant, tests were done for different set points and the results analyzed using an algorithm. The script made determined the overshoot and settling time for each run and compared them graphically. Finally, it was determined the effect of each modeling in the resulting controller response.
\end{abstract}

Keywords: Controle. Modulo experimental de controle. DAQ. Controle PI

\section{Introdução}

A área de Controle é de grande importância na sociedade moderna, sendo presente em diversos processos que requerem garantias de funcionamento e eficiência. Tais garantias devem ser providas pela rigorosa teoria por trás de um sistema de controle e a os componentes fenomenológicos usados na modelagem de processos. Para melhorar o ensino em nível de graduação e proporcionar um maior domínio da teoria e aplicação de controle é comum buscar abordagens que contextualizam a teoria. Dentre as abordagens que incluem práticas destacam-se as project-based, onde alunos precisam desenvolver projetos aplicando os conceitos aprendidos, e lab-based, onde plataformas experimentais sejam usadas em práticas sobre o conteúdo teórico[1].

Uma abordagem prática deve incluir diversos tópicos da teoria e, também, problemas encontrados na implementação de sistemas de controle clássicos, tais como não-linearidades, variações com o tempo e tempo morto. Além do que, mesmo com as muitas plataformas comerciais existentes, a aquisição de equipamentos para um laboratório de controle pode ser muito onerosa em especial quando associada a softwares específicos que requerem licenças anuais. Portanto, alguns projetos buscam juntar as duas abordagens para alunos desenvolverem módulos experimentais de design aberto que futuramente serão usados em práticas por alunos subsequentes. Tais iniciativas usam CLPs, microcontroladores e placas de aquisição de dados (DAQ) de baixo custo e tentam integrar os produtos gerados com programas de licenças abertas ou comumente encontrados em laboratórios de informática de cursos de engenharia para atingir custos mínimos [2-5].

A necessidade de um ensino prático de controle se dá também devido as necessidades da indústria moderna, onde cada vez mais os processos são altamente integrados e há uma grande 
exigência por segurança e eficiência. Em processos industriais atuais, em especial os contínuos, sistemas de controle ineficientes podem levar a propagação de perturbações e perdas. E sistemas não controlados expõem trabalhadores a condições de risco que poderiam ser evitadas [6]. A seguir, serão descritos a planta experimental montada, o posicionamento dos sensores e atuadores, as modelagens aplicadas à planta e a definição usada para cada figura de mérito (sobressinal e tempo de assentamento) no algoritmo de análise.

\section{Materiais e Métodos}

Nesta linha, o trabalho realizado visou a construção de um módulo experimental, no qual se possa aplicar conceitos de controle, de baixíssimo custo e baseado em programas código aberto, resultando na planta da Figura 1a, com os sensores e atuador dispostos como na Figura $1 \mathrm{~b}$. Seguido de duas modelagens, uma fenomenológica e outra caixa-preta, e projeto de um controlador PI para o sistema. Por fim, fizeram-se testes automatizados para a obtenção de figuras de valor e comparação das modelagens e controladores desenvolvidos no trabalho. Além das práticas para obtenção dos resultados apresentados, também é apresentado o critério usado no algoritmo de análise, para identificação de sobre-elevação e tempo de estabilização.

A planta experimental construída foi a de um sistema de dois tanques em série sem interação, sendo este um sistema não-linear, de dinâmica lenta e similar a processos industriais [7]. Sendo assim, a mesma pode mostrar a alunos técnicas de linearização e controle em processo comum no mercado de trabalho. Para a construção do módulo, foi usado em sua maioria materiais reciclados, como garrafas, madeira e mangueiras e outros de fácil acesso como bombas de aquário e sensores de pressão. A placa de circuito para condicionamento de sinais foi feita com componentes de ampla disponibilidade e a DAQ usada foi a USB-DUX, que é open-hardware. O custo total, materiais e DAQ, foi de aproximadamente $\mathrm{R} \$ 360,00$.

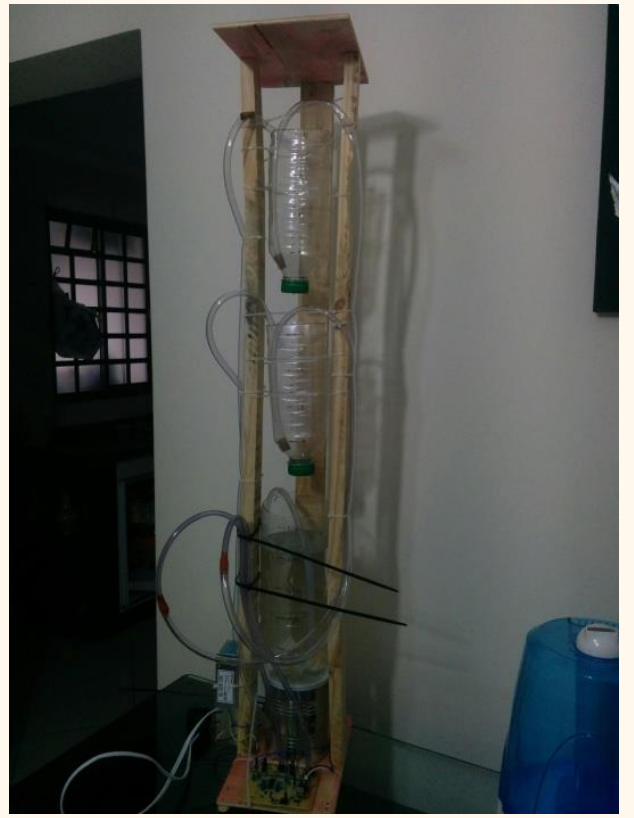

Figura 1a - Foto da planta experimental

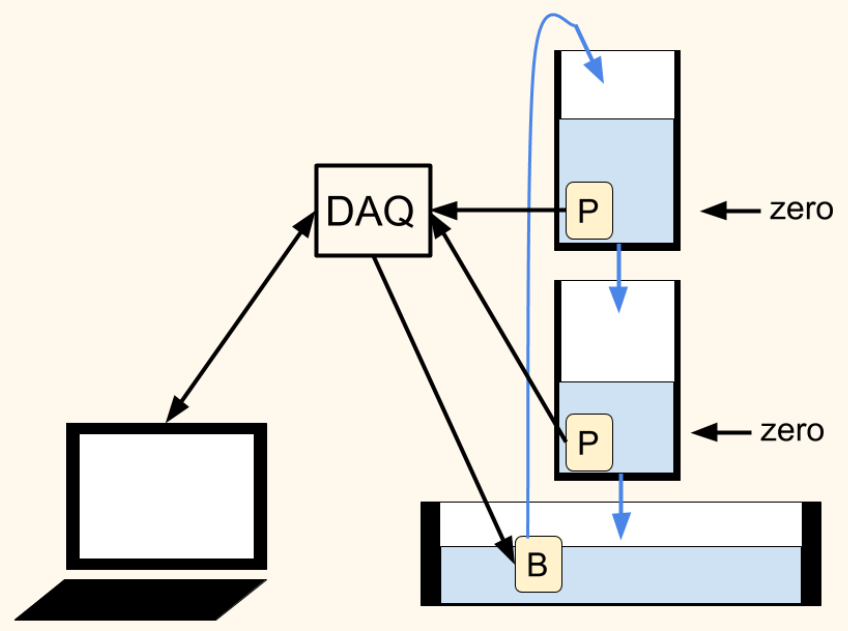

Figura 1b - Diagrama representando o posicionamento dos sensores de pressão $(\mathrm{P}), \mathrm{o}$ atuador (B) e a altura de referência (zero). 
Uma vez montada a plataforma e os subsistemas de condicionamento de sinais e acionamento dos atuadores, fez-se a modelagem fenomenológica. Para tanto, aplicou-se o princípio de Bernoulli levando em conta que os tanques estão sob pressão atmosférica e a resistência dos orifícios de saída é zero, resultando na Equação 1. Então, a equação obtida é linearizada e obtémse a função de transferência presente na Equação 2, onde $A_{s}$ é a área do orifício de saída, $A_{t}$ a área transversal do tanque cilíndrico e $h_{0}$ a altura em que se fez a linearização.

$$
\begin{gathered}
Q=A \sqrt{2 g h} \\
H=\left(s+\frac{\sqrt{g} A_{s}}{\sqrt{2 h_{0}} A_{t}}\right)^{-1}
\end{gathered}
$$

Desta forma, pode-se obter o modelo tomando medidas físicas da planta. Em especial das diversas tampas usadas nos tanques, cada qual possuindo um orifício de tamanho diversos, para gerar variações no modelo.

Subsequentemente, foi realizada a modelagem caixa-preta, na qual, o orifício do tanque foi selado e o tanque foi completado até a marca de $15 \mathrm{~cm}$ para então ter a curva de esvaziamento gravada pela DAQ e processada no software Octave, obtendo o modelo ARX e aproximando para um de sistema de primeira ordem. Uma vez obtidos os polos, foram sintonizados controladores PI. Sendo que, o controle somente do tanque superior foi feito com uma das tampa de menor diâmetro, para evitar que a vazão proveniente do mesmo transborde o segundo tanque. E para o controle do segundo tanque, usou-se uma tampa de orifício maior no superior e a de menor no inferior.

Uma vez realizados experimentos para observar a resposta transitória de cada controlador para diferentes valores de referência, criou-se um algoritmo para análise automática dos dados. Este determina o valor de sobre-elevação e tempo de assentamento e gera um gráfico para cada combinação controlador testado, seja do sistema de um ou dois tanques. Para tanto, considerou-se a sobre-elevação o maior valor lido do sensor de nível dividido pela referência e o tempo de estabilização foi determinado pelo tempo que o valor do sensor de nível leva para entrar, pela última vez, na faixa entre $105 \%$ e $95 \%$ do valor médio dos últimos 10 segundos.

\section{Resultados e Discussão}

A seção seguinte apresenta os resultados obtidos nas duas modelagens realizadas e o desempenho de cada controlador testado. As modelagens foram feitas para o conjunto do sistema de um tanque com uma dada tampa, modelando-os de suas dimensões físicas ou a resposta do sistema a um sinal externo. Sendo que, foram sintonizados controladores PI para o sistema de um tanque (somente o superior) e dois tanques sem interação, e para cada sistema baseou-se o controlador ou no modelo fenomenológico ou no modelo caixa-preta obtido.

Usando a Equação 2 e medidas do tanque e orifícios de suas possíveis tampas, calculou-se o polo do sistema de primeira ordem, obtendo assim a modelagem fenomenológica. Fez-se então a modelagem caixa-preta, também aproximado como um de sistema de primeira ordem, na qual o 
procedimento descrito na seção anterior resultou em outro conjunto de polos. A comparação dos polos obtidos em cada modelagem para cada tampa, nomeadas de A a J, pode ser vista na Figura 2. Nela, os polos obtidos pelo modelo caixa-preta foram, em geral, cinco vezes maiores que os equivalentes no modelo fenomenológico e a correlação é de 0,46683 .

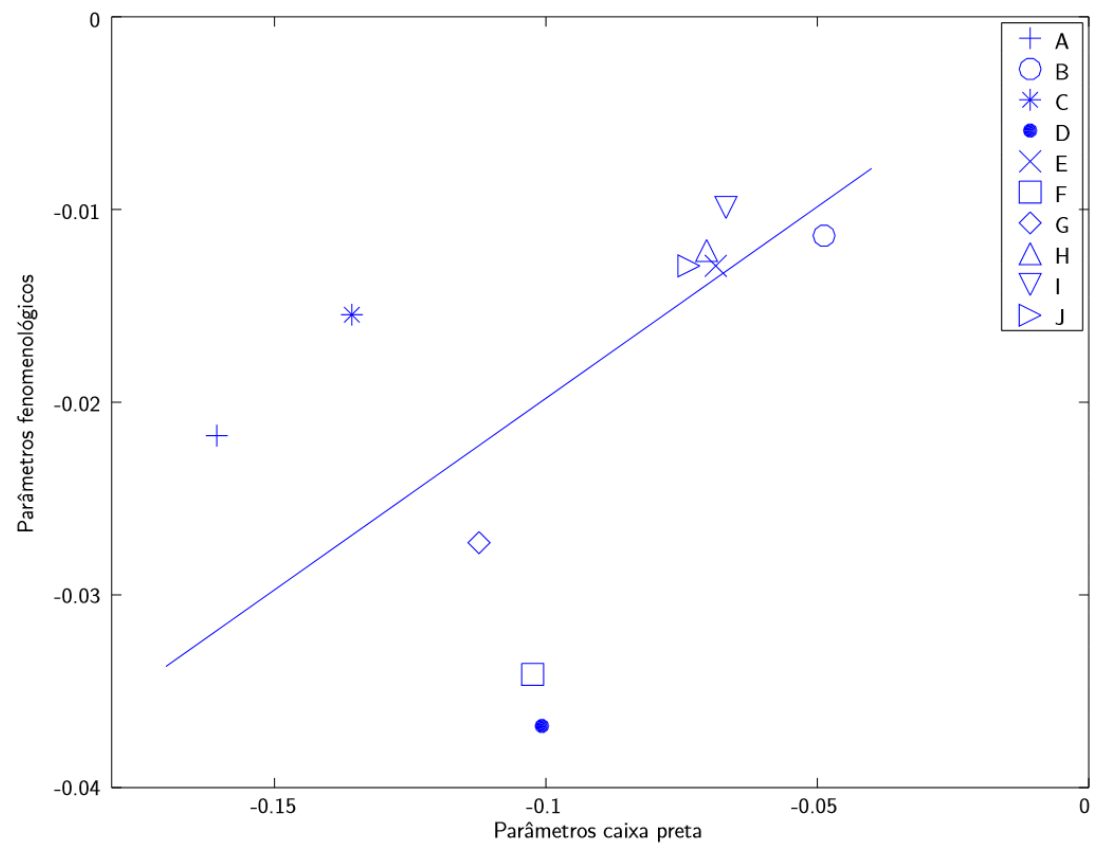

Figura 2 - Polos do sistema de primeira ordem obtido por meio de diferentes modelagens.

Para a comparação dos controladores projetados, que usaram de base os modelos obtidos, usouse o algoritmo descrito anteriormente e foram obtidos gráficos que comparam o tempo de estabilização e sobre-elevação para diversos níveis de referência. Nas Figuras 3a e 3b é possível observar os controladores PI para o tanque superior (sistema com somente um tanque), sendo a primeira figura sobre o controlador baseado no modelo fenomenológico e a seguinte no modelo caixa-preta. As figuras apontam que o controlador baseado em caixa-preta obteve menor tempo de estabilização, entretanto, obteve sobressinal maior nos casos testados. Devido ao número de testes realizados, foi usada a análise automática focando nos parâmetros de sobressinal e tempo de assentamento. As curvas obtidas para cada rodada experimentais se assemelham as apresentadas na Figura 4a e 4b, que contém a resposta para o sistema buscando o valor de referência de $5 \mathrm{~cm}$ (onde valores negativos representam níveis da coluna de água abaixo do nível escolhido como referência para o zero), sendo a primeira referente ao controlador baseado no modelo fenomenológico e o segundo no caixa preta. 


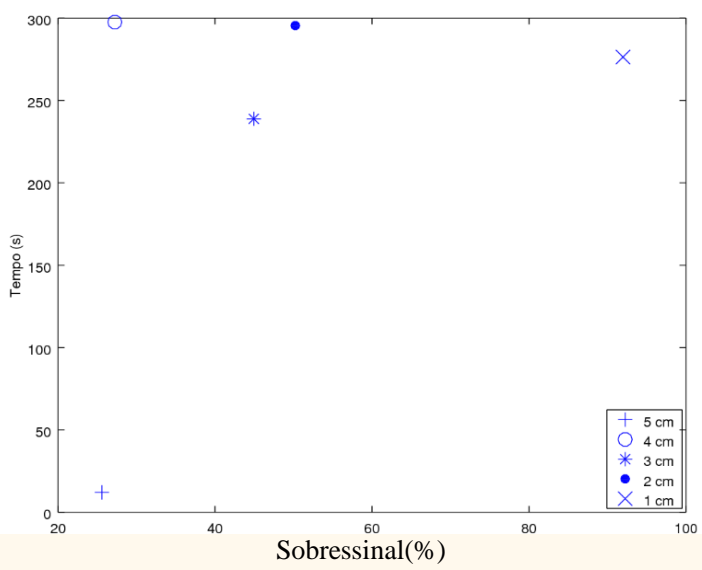

Figura 3a: Métricas para o controlador PI de um tanque, baseado no modelo fenomenológico.

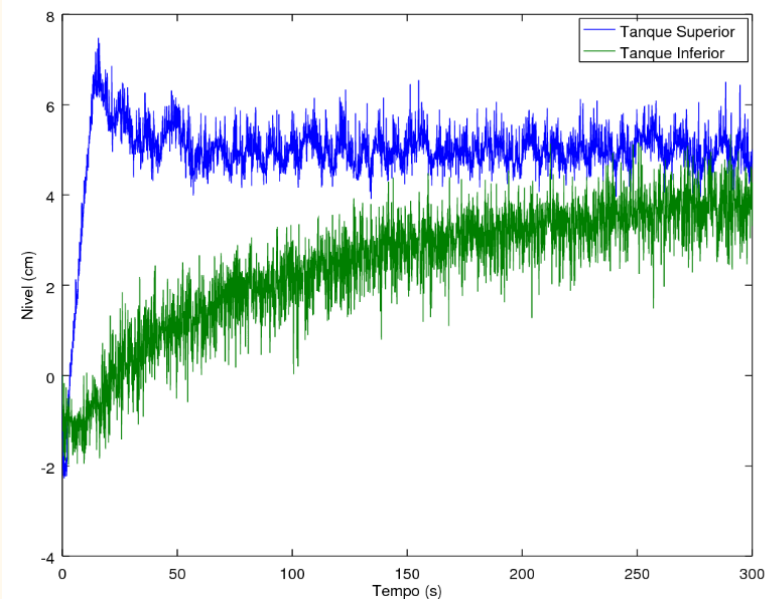

Figura 4a: Resposta do controle baseado no modelo fenomenológico.

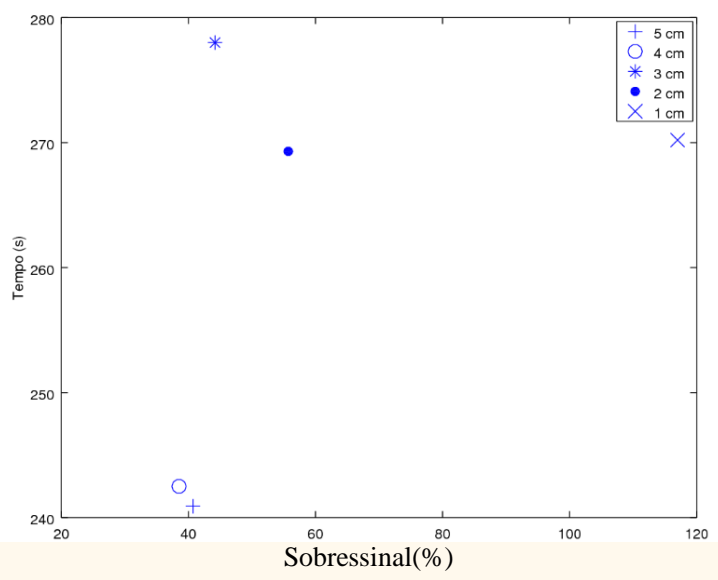

Figura 3b: Métricas para o controlador PI de um tanque, baseado no modelo caixa preta.

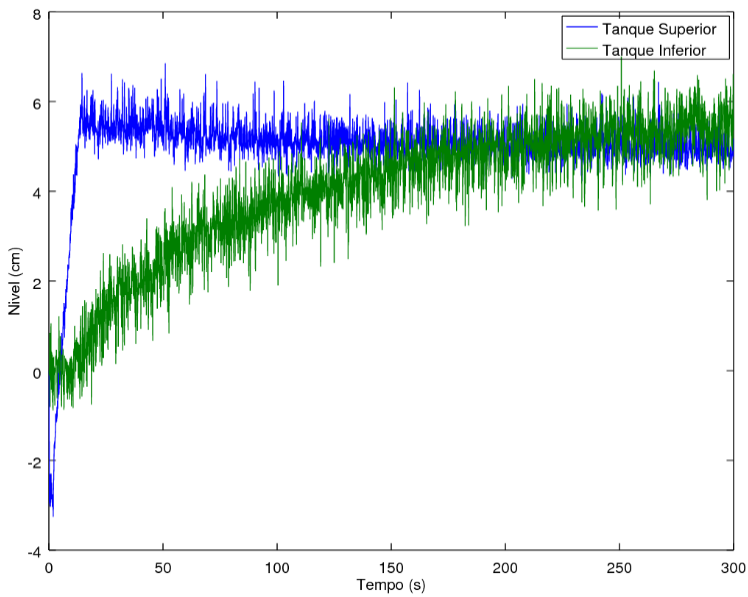

Figura 4b: Resposta do controle baseado no modelo caixa preta.

Foi feita a mesma análise para o sistema de dois tanques sem interação, obtendo as Figuras 5a e 5b. Os controladores apresentaram resultados similares ao caso anterior, em que o menor tempo de estabilização é dos controladores baseados no modelo de caixa-preta, mas o mesmo possui maior sobre-elevação. É notável que a sobre-elevação neste caso foi maior que anterior, em média 3,5 vezes maior. Já a média do tempo de estabilização, para o controlador baseado no modelo caixa-preta houve redução de $12 \%$ e para o baseado no modelo fenomenológico um aumento de $19 \%$. 


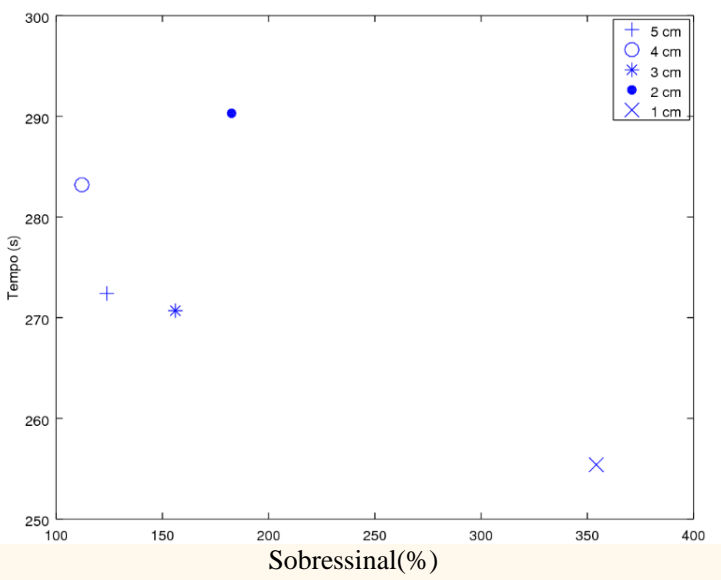

Figura 5a: Métricas para o controlador PI de dois tanques sem interação, baseado no modelo fenomenológico.

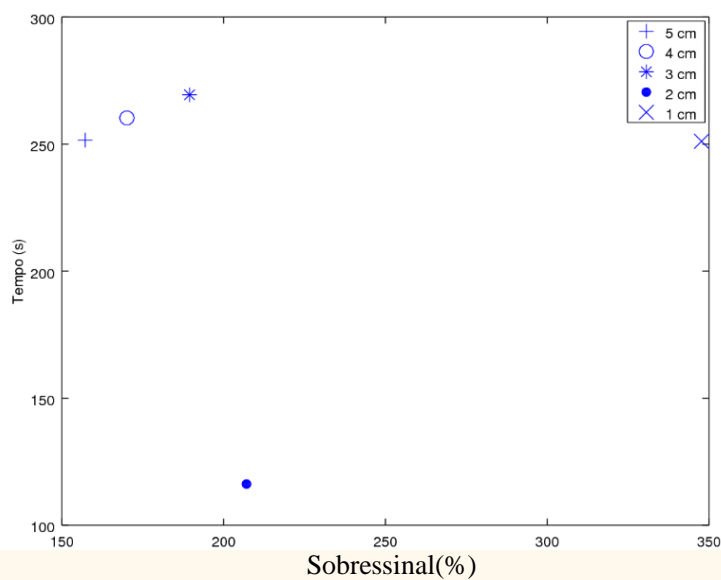

Figura 5b: Métricas para o controlador PI de dois tanques sem interação, baseado no modelo caixa preta.

\section{Conclusão}

O trabalho desenvolvido resultou em um módulo experimental de sistema de controle, de uma planta de controle de nível em um sistema de dois tanques sem interação. Também foram testadas diferentes formas de modelar a planta e controladores PI baseados nos modelos obtidos. O sistema, no entanto, apresenta erros nos sensores de pressão e histerese no atuador, que foram tratados pelo software na DAQ.

Quanto ao desempenho dos controladores testados, o modelo fenomenológico apresentou menor sobre-elevação, mas ambos apresentaram tempos de estabilização similares. Destaca-se que, devido a não haver um atuador capaz de retirar água dos tanques, o sistema apresenta dificuldades em corrigir a sobreatuação. No entanto, o módulo é capaz de ser usado para práticas de controle e apresentar até mesmo conceitos como filtragem de erros, calibração de sensores e atuadores, linearização de sistemas e modelagem caixa-preta.

\section{Referências}

1. AUSLANDER, D.; FRANKLIN, G. F.; CHEOK, K. C.; ASTROM, K. J.; KHEIR, N. A. Control Systems Engineering Education, Automatica, 32, 2. 1996

2. GOMES, F. J.; BALDIOTI, H. R.; GAMA, V. A.; QUEIROZ, F. P. Módulo laboratorial de baixo custo, baseado em foss, para educação em engenharia de controle de processos industriais. in: CLAGTEE. 2011.

3. COCOTA, J. A. N. J.; MONTEIRO, P. M. B.; VIANA, L. M.; MEIRELES, L. V.; COSTA, R. R.; PEIXOTO, A. J. O sistema de controle de nível de tanques no ensino de graduação. In: XI Congreso de Tecnologa, Aprendizaje y Enseñanza de la Electronica, 2014, p. $486-492$. 
4. QUEIROZ, F. P.; FREITAS, L. P. D.; GAMA, V. A.; GOMES, F. J. Desenvolvimento de uma plataforma HILS para educação em controle de processos baseada em FOSS. In: XVIII Congresso Brasileiro de Automática. 2010.

5. SANTOS, C. M. M. dos; COSTA, B. L. G.; SILVA, R. A.; SCALASSARA, P. R. Desenvolvimento de um módulo de controle de nível utilizando o kit Arduino Uno. Anais do XX Congresso Brasileiro de Automática, Setembro 2014, p. 4091-4098.

6. SEBORG, D; MELliCHAMP, .D; Edgar, T.F. Process Dynamics \& Control, 2nd ed, Wiley, Hoboken, 2006.

7. GOSMANN, H. L. Um Sistema Multivariável De Tanques Acoplados Para Avaliação De Técnicas De Controle, MESTRADO. UNIVERSIDADE DE BRASÍLIA, 2002. 